\title{
Tolerable exercise intensity in the early rehabilitation of paraplegic patients. A preliminary study
}

\author{
Paolo Capodaglio, Cesare Grilli and Giacomo Bazzini \\ Ergonomics Unit, Rehabilitation Center of Montescano, 'Clinica del Lavoro' Foundation, 27040 Montescano \\ $(P V)$, Italy
}

\begin{abstract}
Accessible indicators for setting exertion levels in newly injured paraplegias could be useful to improve their daily and occupational performances in less time than is presently required. Eight male newly injured paraplegic subjects performed progressive resisted and endurance exercise tests on an arm cranking ergometer. Cardiorespiratory parameters during exercise were monitored with an oxygen uptake analyzer and perceived exertion was rated on Borg's 10-point scale. Four subjects (Group A) underwent an 'enhanced' rehabilitation protocol which included aerobic arm training sessions. The prescription of exercise intensity was tailored to each subject's tolerable power output for prolonged exertion. The remaining four (Group B) followed a 'conventional' rehabilitation program. Tests were repeated in both groups after 6 weeks. We observed a greater improvement in endurance capacity in Group A than in Group B after completion of the rehabilitation program. A 6-week 'enhanced' rehabilitation program based on a 'moderate' intensity of exercise was well tolerated and effective in improving the fitness levels of newly injured paraplegic subjects. Subjective perception has been shown to be a simple and accessible indicator for setting exertion levels.
\end{abstract}

Keywords: paraplegia; physical work capacity; oxygen consumption; arm cranking; perceived exertion

\section{Introduction}

As a consequence of lower body dysfunction, paraplegic subjects rely on the functional capacity of their arm and trunk muscles in order to perform most activities of daily living. Many of the daily activities of wheelchair dependent persons involve short lasting peak strains and have been recognized as sources of local muscle fatigue and musculoskeletal injuries. ${ }^{1}$ Janssen et $a l^{2}$ reported that such activities place noteworthy demands on the individual anaerobic capacity. Smaller muscle mass recruitment may lead to physical stress and discomfort during activities such as wheelchair propulsion, making transfers and propelling up an incline. ${ }^{3,4}$ Muscle fatigue and musculoskeletal symptoms are often experienced by paraplegic patients during primary rehabilitation and may well prolong their stay in hospital. ${ }^{1}$ High levels of physical strain during certain daily activities may also overexert the cardiovascular system.

In the rehabilitation of patients with recent spinal cord injuries, great emphasis is usually placed on muscle strength training as the means to promote functional independence. Improvements in physical capacity are accompanied by greater freedom of

Correspondence: Dr Paolo Capodaglio, MD mobility and improved occupational performances. Moreover, greater ability to perform daily activities also has favorable psychological effects. ${ }^{5}$

Several authors have reported that the daily activities of wheelchair-confined individuals are insufficient to increase cardiovascular fitness levels. ${ }^{6-9}$ The foregoing studies demonstrated a significant deficit in aerobic capacity for arm crank testing. It was also observed that these deficits were not corrected after a traditional rehabilitation program emphasizing strength training. Consequently, there has been a growing awareness of the importance of assessing the submaximal endurance and of tailoring the training program to the specific needs of paraplegic subjects. Different conditioning training protocols have been proposed. ${ }^{10-12}$

Unfortunately, patients do not always perform at the level one might predict on the basis of clinical tests. $^{13}$ The physical demands of rehabilitation exercises affect psychological motivation, fatigue and satisfaction and, conversely, the patient uses psychological cues to regulate the physiological exercise pace. Thus motivational factors and perception of effort play an important role in determining a patient's capacity to sustain physical effort. ${ }^{14}$ Subjective perceptions of exertion based on Borg's scale ${ }^{15}$ have been found to correlate positively with work load and heart rate for 
different types of activities in healthy subjects ${ }^{16,17}$ and wheelchair users. ${ }^{18}$ We now have a considerable body of literature on the physical work capacity of paraplegic subjects long after injury, ${ }^{9,12,19-24}$ but very few data regarding the responses of newly injured paraplegic subjects to prolonged exertion at low intensities. $^{8,25}$

The aims of this preliminary study were two-fold. Firstly, to assess the physical work capacity and the functional profile of newly injured paraplegic patients before and after completing a 6-week 'conventional' rehabilitation program and a 6-week 'enhanced' rehabilitation program which emphasized the aerobic conditioning. Secondly, to evaluate if the subjective rating of perceived exertion could be used as a reliable indicator of the conditioning levels of exercise.

\section{Methods}

\section{Subjects}

Eight men with complete traumatic spinal cord lesions between T6 and T8 (Table 1) participated in the study. The average interval between injury and the start of the study was 3.2 months. None of the subjects had decubitus ulcers or other major medical problems. Most subjects were taking medications, none of which was expected to significantly interfere with their exercise response.

\section{Instruments}

Subjects were assessed before and after the 'conventional' and the 'enhanced' 6-week rehabilitation program with the following tests: (1) physical work capacity was tested using a mechanically braked arm cranking ergometer (ACE) (Model 881E, manufactured by Monark-Crescent, AB, S-43282, Varberg, Sweden); (2) individual endurance capacity was tested performing prolonged exercises with the ACE; (3) functional status was evaluated using a standardized functional scale (VFM, Valutazione Funzionale per Medullolesi). ${ }^{26}$

During the ergometric tests subjects rated their subjective perception of effort on Borg's 10-point scale. ${ }^{15}$ This simple category scale (Table 2) was

Table 1 Subject's physical characteristics

\begin{tabular}{lccc}
\hline Subjects & Age $($ yrs $)$ & Height $(\mathrm{cm})$ & Body \\
\hline$\# 1$ & 35 & 180 & 75 \\
$\# 2$ & 19 & 185 & 90 \\
$\# 3$ & 45 & 178 & 78 \\
$\# 4$ & 28 & 168 & 70 \\
$\# 5$ & 25 & 165 & 68 \\
$\# 6$ & 24 & 178 & 77 \\
$\# 7$ & 40 & 182 & 88 \\
$\# 8$ & 30 & 180 & 90 \\
mean & 30.7 & 177 & 79.5 \\
\hline
\end{tabular}

developed for differential use and has all the advantages of a general-ratio scale. Numbers are accompanied by readily understood verbal expressions to provide more accurate descriptions of peripheral effort sensations such as aches and pains. The use of verbal expressions makes it possible to quantify the curvilinear variables such as lactate production, especially during intense exercise. The ratio-category scale was developed so that the verbal expressions and perceptual intensity would increase according to a power function. In our study the subjective perception of effort included breathing difficulties and soreness of the arm muscles.

The VFM scale was developed for the functional evaluations of post-acute spinal cord injured patients. The scale measures the dependence/independence level of the paraplegic patient in functional areas related to the activities of daily living. The functional evaluation has to be performed as soon as the patient is able to sit upright in a wheelchair. The VFM score is a composite of eight subsections covering the following activities: eating (6 tests), grooming (7 tests), dressing (8 tests), wheelchair use (15 tests), social skills (9 tests), standing (4 tests) or locomotion ( 7 tests), postural changes ( 5 tests), transfer activities (12 tests). The score for each test ranges from 0 (total dependence) to 4 (total independence). The expected maximum score is therefore 292 .

Respiratory and oxygen uptake variables were measured by a metabolic analyzer (Model EOS Spring, manufactured by Jaeger $\mathrm{GmbH}$, Höchberg, Germany). To determine the physical strain experienced in the hospital setting while performing daily activities, including the rehabilitation sessions, the subjects' $6 \mathrm{~h}$ heart rates (HR) were monitored continuously from 10 am to $4 \mathrm{pm}$ with a Sport Tester wireless monitor (Model PE3000, manufactured by Polar Electro Ky, Kempele, Finland). Individual data were collected over 3 days and the mean HR profile was considered. Variance in daily activity schedules of each of the eight patients was minimized by giving each patient the same therapy schedule. The energy

Table 2 Borg's 10-point Category-Ratio (CR-10) scale. The numerical score is 'anchored' to simple verbal expressions

\begin{tabular}{cc}
0 & Nothing at all \\
0.5 & Very, very weak (just noticeable) \\
1 & Very weak \\
2 & Weak \\
3 & Moderate \\
4 & Somewhat strong \\
5 & Strong \\
6 & Very strong \\
7 & \\
8 & \\
9 & Very, very strong (almost maximal) \\
10 & Maximal \\
\hline
\end{tabular}


expenditure of the paraplegic patients during daily activities and rehabilitation sessions was estimated from the individual linear relationship between $\mathrm{HR}$ and oxygen consumption $\left(\dot{\mathrm{VO}}_{2}\right)$ at submaximal intensities. We referred the mean $6 \mathrm{~h} \mathrm{HR}$ to the individual's regression equation between $\mathrm{VO}_{2}$ and $\mathrm{HR}$ obtained during the ACE testing.

The arm cranking unit was secured to an adjustable table in order to keep the shoulder in line with the axis of the ACE. The wheelchair was placed so that the subject's arm was fully extended when the pedal was at its greatest distance. A speedometer was observed by the subject to sustain the prescribed velocity $(50 \mathrm{rpm})$.

\section{Procedure}

Tests Testing sessions were started as soon as the subjects were able to sit upright in a wheelchair. Subjects were first familiarized with the technique for grading perceived exertion on Borg's 10-point scale. All tests were performed at least $2 \mathrm{~h}$ after the mealtimes.

Each subject first performed a progressive resisted arm cranking exercise test. The test started with a 3 min warm-up at $0 \mathrm{Watt}$, followed by increments of 12.5 Watts every 2 min until subjects rated the exertion as 'maximal'. The following parameters were monitored every $30 \mathrm{~s}$ : $\mathrm{HR}, \mathrm{VO}_{2}$, ventilation (VE) and respiratory exchange ratio (RER). The electrocardiogram was monitored throughout the testing sessions.

Each subject then performed, in separate sessions over a 4 day period, a total of eight $15 \mathrm{~min}$ endurance tests with the ACE at two constant work loads corresponding to the perceived exertion ratings of 3 ('moderate' effort) and 4 ('somewhat strong') on Borg's scale obtained in the incremental test. The order of load presentation was randomized. Monitoring procedures and criteria for interrupting the exertion were the same as those used in the incremental test. These further endurance tests were performed to monitor the growth over time of perceived exertion parallel to the increase of physiolgic parameters and to allow the subsequent analysis of the 'iso-perception' curves.

Rehabilitation Four subjects underwent an 'enhanced' rehabilitation program (Group A). The other four subjects underwent the 'conventional' rehabilitation program used in our Center (Group B). The 'enhanced' rehabilitation program consisted of 20 to $30 \mathrm{~min}$ of arm cranking per day in addition to the 'conventional' program. The intensity of the exercise corresponded to the subjective tolerable power output for prolonged exertion obtained from the progressive resisted tests with the ACE ('moderate' subjective perception of effort and RER $<1$ ). The 'enhanced' rehabilitation program is presented in Table 3 . The program aims to increase strength and endurance by varying the duration, the level of exercise intensity and the number of revolutions per minute of the arm cranking bouts.

The 'conventional' rehabilitation program consisted of at least $4 \mathrm{~h}$ per day including: strengthening activities $(3 / 4 \mathrm{~h})$, maintaining range of motion and stetching $(1 / 2 \mathrm{~h})$, training in transfers and activities of daily living, wheelchair positioning, standing $(2 \mathrm{~h})$, respiratory training $(1 / 2 \mathrm{~h})$, bowel and bladder management, psychological support. All tests were repeated in both groups after 6 weeks.

\section{Data analysis}

The ratings of perceived exertion given during the endurance tests were analyzed with a statistical software program (StatPak, NWA Analytical Software, Portland, Oregon), which allowed us to compute the least-squares coefficients of a single-variable regression for power functions. The analysis was performed to define the individual 'iso-perception' curves in a power/duration reference system. The 'isopercetion' curve fitted all the individual values of mechanical power output collected during the eight 15 min endurance tests that corresponded to a same perceptual level on Borg's scale. The standard error of estimate (SEE) of the data points sampled during the endurance tests was used to generate a confidence interval around the 'iso-perception' curves obtained.

An independent $t$ test was used to determine whether there were any significant differences between the groups at pretest. Then an independent $t$ test was run on the posttest data to determine whether there was a significant treatment effect. A two-tailed test was performed with alpha set at 0.05. A Wilcoxon Rank Sum Test was chosen to analyze differences in non-

Table 3 The 'enhanced' rehabilitation program (Group A)

\begin{tabular}{llcccc}
\hline \multicolumn{1}{c}{ Target } & $\begin{array}{c}\text { Duration (min) } \\
\text { Day }\end{array}$ & Repetitions & Rpm & Intensity & Intensity (Watt) \\
\hline Mon & Endurance (low intensity) & $5 \times 3$ & 50 & 'Light' & $2.5-5$ \\
Tue & Strength & $3 \times 3$ & $35-40$ & 'High' & 15 \\
Wed & Endurance & $5 \times 3$ & 50 & 'Moderate' & $5-7.5$ \\
Thur & 'Coordination' & $3 \times 5$ & 70 & 'Light' & 0 \\
Fri & Endurance & $5 \times 3$ & 50 & 'Moderate' & $5-7.5$ \\
\hline
\end{tabular}

The targets of the arm cranking exercises had to be obtained by varying the duration, the number revolutions per minute (rpm) and the level of exercise intensity (expressed by the nominal ratings of subjective perception of effort on Borg's scale and by the relative power outputs). 'Coordination' refers to the capacity to maintain a high cranking rate 
parametric scores. The 5\% significance level was used for hypothesis testing.

\section{Results}

Physical work capacity and functional score Individual and group data for pre- and post-training maximal workloads, $\mathrm{V}_{2}$ values and functional scores are presented in Table 4 . No statistically significant differences between groups were found in $\mathrm{VO}_{2}$ and work exerted values at pre-training. Statistically significant $(P<0.05)$ differences in $\mathrm{VO}_{2}$ values were found between groups at post-training. Mean increases of $62.6 \%$ were found in Group A for post-training $\mathrm{VO}_{2}$ values. Differences in work exerted between groups was not statistically significant at post-training.

Non significant differences were found in the mean functional scores (VFM mean total score) between groups at pre- and post-training. The mean VFM score in Group A increased from 185/2192 (range $170-196$ ) to $232 / 292$ (range $212-254$ ). The mean VFM score in Group B increased from 179/292 (range $170-190$ ) to $225 / 292$ (range $210-246$ ).

\section{Daily energy expenditure}

The energy expenditure mean values (Table 5) of the paraplegic subjects performing daily activities, including the rehabilitation sessions, in the hospital setting was obtained by referring the subjects' $6 \mathrm{~h}$ HR values monitored continuously from $10 \mathrm{am}$ to $4 \mathrm{pm}$ to the individual's $\mathrm{VO}_{2} / \mathrm{HR}$ regression equation obtained in the incremental test.

Correlations between subjective and objective parameters monitored

In all eight subjects the ratings of perceived exertion during the incremental test correlated significantly with mechanical work $(r=0.9, P<0.001)$, the $\dot{\mathrm{VO}}_{2}$ values $(r=0.98, P<0.001)$ and the percentage of maximum HR as calculated by the formula $220-$ age $(r=0.98$, $P<0.001$ ).

Correlations between the mean $\mathrm{HR}$ values and the mean perceived exertion scores obtained during the endurance tests at different workloads were statistically significant in all eight subjects. Linear correlations between the two measures ranged from 0.59 to 0.88 during the endurance tests at a level of perception of 3 ('moderate' effort) and from 0.81 to 0.9 at a level of perception of 4 on Borg's scale.

\section{Changes in endurance capacity}

Regression analysis was performed to determine the individual 'iso-perception' curves obtained from the experimental data points sampled during the endurance tests. The curves were represented by the power functions; $Y=c . \quad X^{n}, \quad(-1<n<0)$, describing the relationship between power output and duration. Improvement in endurance capacity after the 6 week 'enhanced' training program at a 'moderate' (score 3) intensity level was monitored by the shifting of the

Table 5 Mean and peak daily energy expenditure values of the eight subjects estimated from the individual's $6 \mathrm{~h} \mathrm{HR}$ monitored from $10 \mathrm{am}$ to $4 \mathrm{pm}$ and referred to the individual $\mathrm{VO}_{2} / \mathrm{HR}$ regression equation obtained in the incremental test with the ACE

\begin{tabular}{lcccc}
\hline Subject & Mean $\mathrm{HR}$ & Mean $\mathrm{VO}_{2}$ & Peak $\mathrm{HR}$ & Peak $\mathrm{VO}_{2}$ \\
\cline { 3 - 4 }$\# 1$ & 90.3 & 0.47 & 132 & 0.93 \\
$\# 2$ & 105.6 & 0.58 & 160 & 1.1 \\
$\# 3$ & 108 & 0.7 & 158 & 1.3 \\
$\# 4$ & 112 & 0.75 & 172 & 1.6 \\
$\# 5$ & 102 & 0.6 & 140 & 1 \\
$\# 6$ & 95 & 0.5 & 135 & 0.9 \\
$\# 7$ & 104 & 0.7 & 152 & 1.1 \\
$\# 8$ & 105 & 0.65 & 140 & 0.9 \\
\hline
\end{tabular}

Table 4 Raw data, mean and range values for pre- and post-training workloads ( $\mathrm{kpm})$ and $\mathrm{VO}_{2} \mathrm{values}\left(\mathrm{ml} \mathrm{kg}^{-1} \mathrm{~m}^{-1}\right) \mathrm{during}$ the progressive resisted arm cranking test and functional scores (VFM) of the eight newly injured paraplegic subjects (1-4 Group A; 5-8 Groups B). Statistically significant differences between the groups were found in $\mathrm{VO}_{2}$ values at post-training

\begin{tabular}{|c|c|c|c|c|c|c|}
\hline \multirow[b]{2}{*}{ Subject } & \multicolumn{2}{|c|}{ 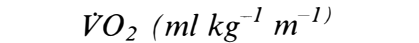 } & \multicolumn{2}{|c|}{ Workload (Kpm) } & \multicolumn{2}{|c|}{ VFM score } \\
\hline & Pre & Post & Pre & Post & Pre & Post \\
\hline$\# 1$ & 10.9 & 21.7 & 675 & 900 & 183 & 220 \\
\hline$\# 2$ & 9.7 & 19.2 & 562 & 900 & 170 & 212 \\
\hline$\# 3$ & 13.4 & 24.1 & 750 & 1050 & 191 & 245 \\
\hline$\# 4$ & 16.7 & 27.6 & 800 & 1500 & 196 & 254 \\
\hline mean & 12.6 & 23.1 & 696.7 & 1087.5 & 185 & 232 \\
\hline range & $(9.7-16.7)$ & $(19.2-27.6)$ & $(562-800)$ & $(900-1050)$ & $(170-196)$ & $(212-254)$ \\
\hline \#5 & 9.4 & 12.3 & 550 & 675 & 170 & 210 \\
\hline$\# 6$ & 11 & 15.6 & 675 & 850 & 185 & 230 \\
\hline \#7 & 13.2 & 16.4 & 800 & 900 & 190 & 246 \\
\hline \#8 & 10.5 & 14.7 & 675 & 800 & 174 & 216 \\
\hline mean & 11 & 14.7 & 675 & 806.2 & 179.7 & 225.5 \\
\hline range & $(9.4-13.2)$ & $(12.3-16.4)$ & $(550-800)$ & $(675-900)$ & $(170-190)$ & $(210-246)$ \\
\hline
\end{tabular}


individual 'iso-perception' curves corresponding to a perception of 'moderate' effort toward higher power output levels in Group A (Figure 1). The SEE of the experimental data points samples in the pre-training endurance tests was used to generate a confidence interval around the pre-training 'iso-perception' curves (Table 6). To define 'significant' post-training differences, we calculated if the post-training individual values were within or beyond the confidence intervals of the pre-training 'iso-perception' curves ('moderate' effort). The post-training curves in Group A were

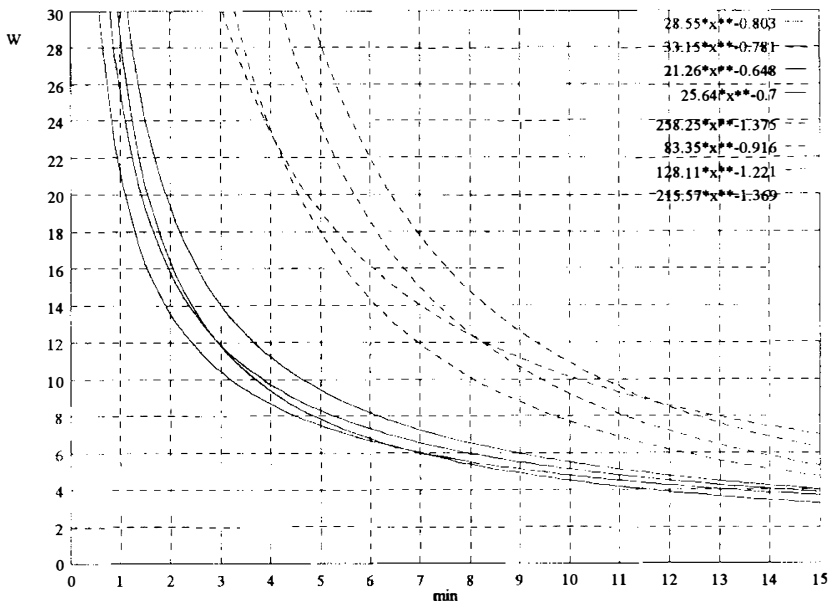

Figure 1 The individual 'iso-perception' curves ('moderate' effort) obtained from the experimental data points sampled during the endurance tests were represented by power functions; $Y=c . X^{n},(-1<n<0)$, describing the relationship between power output and duration. (The equations of the pre- and post-training power functions are reported in the top right-hand corner). Endurance capacity was monitored in the four patients undergoing the 'enhanced' rehabilitation program (Group A) by the trend of the 'iso-perception' curves. The improvements in endurance capacity after the 6 week training program at 'moderate' intensity level are shown by the shifting of the post-training individual 'iso-perception' curves (dashed lines) toward higher output levels in all the patients tested

Table 6 The table represents the equations of the eight individual pre-training 'iso-perception' curves and the SEE of the values sampled during the endurance tests and fitted by the same curves. The SEE was used to generate a confidence interval around the pre-training curve

\begin{tabular}{ll}
\hline Curve equations & $S E E$ \\
\hline$y-28.55 \times^{-0.803}$ & 0.14 \\
$y-33.15 \times 0.781$ & 0.19 \\
$y-21.26 \times^{-0.648}$ & 0.16 \\
$y-26.64 \times 0.7$ & 0.17 \\
$y-29 \times^{-0.805}$ & 0.21 \\
$y-35.2 \times^{-0.74}$ & 0.19 \\
$y-23.8 \times^{-0.73}$ & 0.15 \\
$y-20.9 \times^{-0.6}$ & 0.2 \\
\hline
\end{tabular}

shifted far beyond the confidence limits generated by the SEE of the pre-training experimental data points. This finding was not reported in Group B (Figure 2).

None of the eight subjects exceeded an RER value of 1 during the endurance tests at a constant perception of 'moderate' effort.

\section{Discussion}

Circulatory load imposed by daily activities can be roughly estimated as the product of heart rate, mean blood pressure and stroke volume and is largely dependent upon the intensity and type of muscular work. The quantitative relationships between these parameters are not the same under every exercise condition. ${ }^{27}$ The range of physical activities to which paraplegic subjects are confined is fairly restricted and therefore heart rate monitoring may be considered a reliable method for assessing the circulatory strain during the day. ${ }^{6}$

There is considerable evidence that ACE training has a significant effect on patients with spinal cord lesions who have small muscle masses available for training. ${ }^{12,19,28-30}$ Different views are held as to which criteria are most suitable for setting the intensity of training. There is a general consensus that training is an efficient stimulus only if higher work intensities act for prolonged periods of time. ${ }^{33-35}$ At a theoretical level, the optimal work intensity for endurance training in health subjects should be within the range of the 'aerobic-anaerobic threshold', where a high stimulation

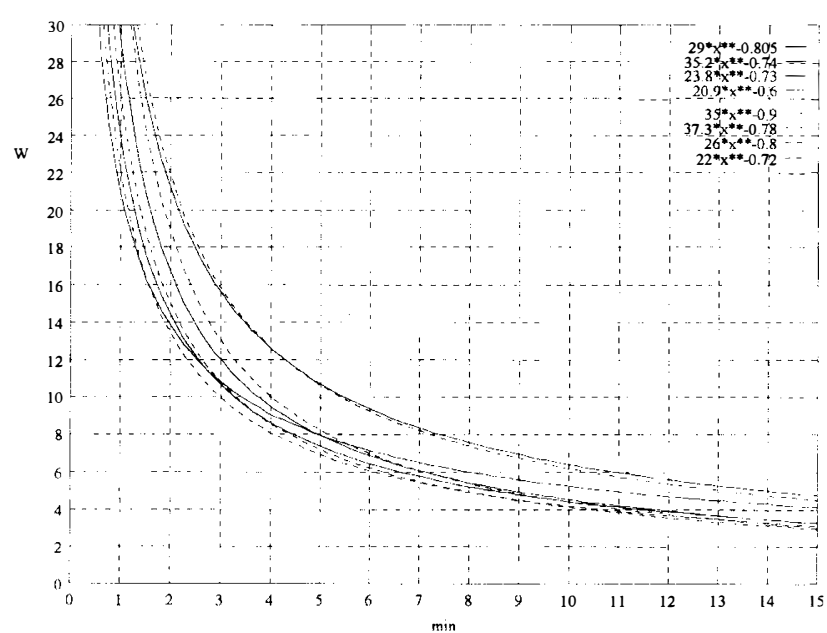

Figure 2 The individual 'iso-perception' curves ('moderate' effort) obtained from the experimental data points sampled during the endurance tests of the four patients undergoing the 'conventional' program (Group B). (The equations of the power functions before and after the rehabilitation program are reported in the top right-hand corner). The experimental data points generating the post-training individual 'isoperception' curves (dashed lines) were within the confidence limits generated by the SEE of the data points fitted by the pre-training individual 'iso-perception' curves (solid lines) 
of the oxidative metabolism in skeletal muscle cells is present, with only little use being made of mechanisms that would lead to lactate production. Empirical data in able-bodies subjects indicate that endurance training will maintain the state of conditioning when performed within the range of the anaerobic threshold. ${ }^{34-36}$ The anaerobic threshold has been suggested as parameter for assessing submaximal endurance and for setting the intensity threshold of training in paraplegics injured for a mean of 6 months. ${ }^{10}$ Jochheim and Strohkendl, ${ }^{11}$ demonstrated that exercise intensities equivalent to $50-60 \%$ of maximal heart rate reserve will contribute to the maintenance of cardiovascular fitness in paraplegic subjects. An early physical therapy program should impose minimal energy demands since spinal cord injured patients are known to manifest marked metabolic depression after injury. ${ }^{24}$ Hooker and Wells, ${ }^{12}$ compared submaximal exercise responses after 8 weeks of wheelchair ergometer training in spinal cord injured subjects and found a significant training effect at a moderate training intensity (10 Watts).

The subjects in the present study had motor complete spinal cord lesions between T6 and T8. It could be argued that with this homogeneous level of disability and motor ability, a similar amount of available and active muscle mass was utilized during both programs. A pertinent finding in our preliminary study was that a 'moderate' level of intensity (score 3 on Borg's 10-point scale) elicited a noticeable training effect in the newly injured paraplegic patients in Group A, as monitored by the shifting of the 'iso-perception' curves toward higher power outputs. No statistical tests were used in our study to support the significance of the shifting of the curves. We did observe, however, that the experimental data points of the shifted curves were far beyond the confidence intervals generated around the pre-training 'iso-perception' curves. We therefore assumed that the individual power output generated at the same perceptual level and time reference was 'significantly' increased after the completion of the 'enhanced' rehabilitation program. The patients who underwent the 'enhanced' rehabilitation program (Group A) showed higher aerobic capacity at discharge than the controls. A limitation of our study is the small sample size, which could have affected the significantivity of the results.

On the basis of our results, however, the individual tolerable intensity of prolonged arm cranking exercise may be defined by the 'iso-perception' curve corresponding to a 'moderate' effort on Borg's scale (score 3). Physiological parameters remained constant at this level of perceptual intensity, with the RER value around 1. Therefore, the area delimited by the individual 'iso-perception' curve ('moderate' effort) may represent the tolerable non-fatiguing power output over time for prolonged arm cranking exercise. This preliminary study emphasizes the role of an aerobic training program in addition to a 'conventional' program in the early rehabilitation of paraplegic subjects and suggests that ratings of perceived exertion during exercise may be a simple yet valuable means of evaluating effective exercise intensity. It also suggests that for compliant patients, exercise intensity should be set on the basis of the subjective perception of effort, after having assessed the individual physical work capacity. Within the limitations of our study, the small differences between the individual ratings of perceived exertion over time appear to vouch for the reliability of self-assessment as an indicator of fatigue in a homogeneous group. Another salient advantage of this approach is that submaximal work intensities are ideally suited to the evaluation of physical work capacity and to the monitoring of progress in physical conditioning programs for newly injured paraplegic subjects. Some scepticism in the literature remains regarding the use of perceived exertion in rehabilitation, ${ }^{37}$ and further research is needed to confirm its reliability in the disabled population. Morgan, ${ }^{38}$ has calculated that most physiological variables account for only $67 \%$ of the total variance in ratings of perceived exertion.

Further research is also needed to determine which training protocol will be the most effective in enabling paraplegic patients to reach the goals of independent living. Future studies should concentrate on exploring different combinations of intensity, duration and frequency and examining their effectiveness.

\section{Acknowledgements}

The Authors wish to thank Gillian Jarvis for her invaluable editorial assistance.

\section{References}

1 Knutsson E, Lewenhaupt-Olsson E, Thorsen M. Physical Work Capacity and Physical Conditioning in Paraplegic Patients. Paraplegia 1973; 11: $205-206$

2 Janssen TWJ et al. Physical strain during activities of daily living in male spinal cord injured subjects: relation to physical performance capacity. In: van der Woude LHV, Meijs PJM, van der Grinten BA, de Boer YA. (eds). Ergonomics of manual wheelchair propulsion: State of the art. Milano: Pro Juventute (1993).

3 Wolfe GA, Waters R, Hislop HJ. Influence of floor surface on the energy cost of wheelchair propulsion. Physical Therapy 1971; 57: $1022-1027$.

4 Glaser RM, Sawka MN, Brune MF, Wilde SW. Physiological responses to maximal effort wheelchair and arm crank ergometry. J Appl Physiol: Respirat Environ Exercise Physiol 1980; 48(6): $1060-1064$.

5 Roberts K. Sport for the disabled. Physiotheraphy 1974; 60: $271-4$.

6 Hjeltnes N, Vokac Z. Circulatory strain in everyday life of paraplegics. Scand J Rehabil Med 1979; 11: 67-73.

7 Figoni SF. Spinal cord injury and maximal aerobic power. $\mathrm{Am}$ Corr Ther $J$ 1984; 38: 44-50.

8 Ellenberg $\mathrm{M}$ et al. Aerobic capacity in early paraplegia: implications for rehabilitation. Paraplegia 1980; 27: 261-268.

9 Eriksson P, Lofstrom L, Ekblom B. Aerobic power during maximal exercise in untrained and well-trained persons with quadriplegia and paraplegia. Scand J Rehab Med 1988; 20: $141-147$. 
10 Lin KH, Lai JS, Kao MJ, Lien IN. Anaerobic threshold and maximal oxygen consumption during arm cranking exercise in paraplegia. Arch Phys Med Rehabil 1993; 74: 515-520.

11 Jochheim KA, Strohkendl $H$. The value of particular sports of wheelchair-disabled in maintaining health of the paraplegic. Paraplegia 1973; 11: 173-178.

12 Hooker SP, Wells CL. Effects of low- and moderate-intensity training in spinal cord-injured persons. Med Sci Sports Exerc 1988; 21(1): $18-22$.

13 Gage M. The appraisal model of coping: An assessment and intervention model for occupational therapy. American Journal of Occupational Therapy 1992; 46: 353 - 362.

14 Gamberale F. Perception of effort in manual materials handling. Scand J Work Envirom Health 1990; 16(1): 59-66.

15 Borg G. A category scale with ratio properties for intermodal and interindividual comparisons. In Geisler HG, Petzold P (eds). Psychophysical judgement and the process of perception. Berlin, Germany: VEB Deutscher Verlag der Wissenschaft, 1982, pp. $25-34$.

16 Gamberale F. Perceived exertion, heart rate, oxygen uptake and blood lactate in different work operations. Erognomics 1972; 15: $545-554$.

17 Ulmer HV, Janz U, Lollgen H. Aspects of the validity of Borg's scale. Is it measuring stress or strain? Wenner-Gren Center International Symposium Series 1977; 28: 181-199.

18 Birk TJ et al. Relationship of perceived exertion and heart rate response during exercise testing in wheelchair users. In: Proceedings of the American Alliance for Health, Physical Education, Recreation and Dance. Minneapolis. 1983.

19 DiCarlo SE, Supp MD, Taylor HC. Effect of Arm Ergometry Training on Physical Work Capacity of Individuals with Spinal Cord Injuries. Physical Therapy 1983; 63 (7): $1104-1107$.

20 Gass GC, Camp EM. The maximum physiological responses during incremental wheelchair and arm cranking exercise in male paraplegics. Med Sci Sports 1984; 16: 355.

21 Nilsson S, Staff D, Pruett E. Physical work capacity and the effect of training on subjects with long standing paraplegia. Scand $J$ Rehab Med 1975; 7: 51-56.

22 Zwiren LD, Bar-Or O. Responses to exercise of paraplegics who differ in conditioning level. Medicine and Science in Sports 1975; 7: $94-98$.
23 Coutts KD, Rhodes EC, McKenzie DC. Maximal exercis responses of tetraplegics and paraplegics. J Appl Physiol 1983 55: 479.

24 Huang $\mathrm{CH}$ et al. Prescriptive arm ergometry to optimiz muscular endurance in acutely injured paraplegic patients. $\mathrm{ArCl}$ Phys Med and Rehabil 1983; 64: 578-582.

25 Borg G. Physical performance and perceived exertion [disserta tion]. Lund (Sweden): University of Lund, 1962.

26 Taricco $\mathrm{M}$ et al. Social and vocational outcomes in patients witl spinal cord injury. Paraplegia 1992; 30: $214-219$

27 Burger GCE. Heart rate and the concept of circulatory load Ergonomics 1969; 12: 857.

28 Pollock ML et al. Arm pedaling as an endurance training regime for the disabled. Arch Phys Med Rehabil 1974; 55: 418 424.

29 Clausen JP, Trap-Jensen T, Lassen NA. Effects of training or heart rate during arm and leg exercises. Scan J Clin Lab Inves 1970; 26: $295-301$

30 Di Carlo SE. Improved cardiopulmonary status after a two month program of graved arm exercise in a patient with $\mathrm{C}$ quadriplegia. Phys Ther 1982; 62: 456-459.

31 Davies CTM, Knibbs AV. The training stimulus. Int $Z$ Anger Physiol 1971; 29: 299-305.

32 Katch V, Weltman A, Sady S, Freedson P. Validity of the relativ percent concept for equating training intensity. Eur J ApP Physiol 1978; 39: 219-227.

33 Shepard RJ. Intensity, duration and frequency of exercise a determinants of the response to a training regime. Int $Z$ Angel Physiol 1968; 26: $272-278$.

34 Kindermann W, Simon G, Keul J. The significance of th aerobic-anaerobic transition for the determination of workloa intensities during endurance training. Eur J Appl Physiol 1979 42: $25-34$

35 Hagberg JM. Physiological implications of the lactate threshold Int J Sports Med 1984; 5(Suppl): 106-109.

36 Yoshida T, Suda Y, Takeuchi M. Endurance training regime based upon arterial blood lactate: effects on anaerobic threshold Eur J Appl Physiol 1982; 49: 223-230.

37 Hage P. Perceived exertion: one measure of exercise intensity. Physician and Sportsmedicine 1981; 9: 136-143.

38 Morgan WP. Psychological factors influencing perceived exertion. Medicine and Science in Sport 1973; 5: 97-103. 